\title{
WAVES OF EXCITATIONS IN HETEROGENEOUS ANNULAR REGION II. STRONG ASYMMETRY
}

\author{
KRISTÓF KÁLY-KULLAI, ANDRÁS VOLFORD and HENRIK FARKAS \\ Department of Chemical Physics \\ Budapest University of Technology and Economics \\ Budapest H-1521 \\ E-mail:kk222@ural2.hszk.bme.hu,volford@phy.bme.hu,farkas@phy.bme.hu
}

\begin{abstract}
Excitation wave propagation in a heterogeneous medium around a circular obstacle is investigated, when the obstacle is located very eccentrically with respect to the interfacial circle separating the slow inner and the fast outer region. Qualitative properties of the permanent wave fronts are described, and the calculated wave forms are presented.
\end{abstract}

1. Introduction. Wave propagation in excitable media is widely studied in chemistry and biology. Such excitation waves are usually described by using reaction-diffusion equations. However, these waves can be treated by geometrical approach too [7]. The first 2D application of this approach goes back to Wiener and Rosenblueth [14] who constructed a model to describe propagation of excitations in cardiac muscle. This model is the base of the kinematic model or geometrical wave theory. By using reaction-diffusion equations the wave fronts are derived from partial differential equations as equiconcentration surfaces. In the geometrical approach the initial front is given and the evolution of the front is defined by the Fermat principle of minimal propagation time.

A travelling wave can be described by a wave function $u$ of the form

$$
u(r, t)=A(r) f(t-S(r)),
$$

where $r$ is the space vector, $t$ denotes the time, $A$ is the amplitude, $f$ is the phase, and $S$ is the eikonal. The wave fronts are defined as level surfaces of the eikonal $S$. The

2000 Mathematics Subject Classification: Primary 46C20; Secondary 32G81.

Key words and phrases: Caustics, Geometrical theory of waves.

The authors are grateful to the funds OTKA T-30110 and T-042708 and Békésy György Postdoctoral Fellowship for supporting this research.

This paper is a continuation of the previous work [12].

The paper is in final form and no version of it will be published elsewhere. 
propagation process can be described without involving amplitude, by using only the basic concepts of rays and fronts. The main advantage of this approach is the pictorial concept. The solutions of the PDEs are hardly visual without further processing, while the usage of rays and fronts in the geometrical wave theory enables us to represent the development of the fronts easily. Furthermore, the computer program [4] based on the geometrical wave theory is faster than the ones used for reaction diffusion equations. On the other hand, the geometrical wave theory accounts for the shapes and evolution of the wave front, but not for such interesting phenomena like curvature effect, wave splitting, chemical turbulence.

Previously the geometrical wave theory was successfully applied to describe chemical waves in non-uniform membrane rings. At first, the symmetric arrangement was studied, basic concepts were introduced and fundamental features were established [10, 5]. Later $[12,13]$, the moderately asymmetric arrangement was examined and new phenomena were recognized.

In this paper we examine the strongly asymmetric arrangement. For this case, some additional features of wave propagation are shown.

2. Terminology and the model. The geometric wave theory is based on Fermat's principle of least propagation time:

$$
\tau(g)=\int_{g} d s / v=\text { minimum }
$$

where $g$ is a curve, $s$ is the arc length, $v$ is velocity of propagation and $\tau$ is the propagation time. This condition specifies the Fermat ray(s) connecting the given points $P_{1}$ and $P_{2}$. Furthermore, for a given initial wave front $f_{0}$ we can define Fermat's rays starting from $f_{0}$. The orthogonal trajectories of rays starting from $f_{0}$ will be the subsequent wave fronts belonging to different instants from the initial front.

Here we follow the terminology and definitions used in [12], but we apply them to the strongly asymmetric arrangement. Let $v_{s}$ and $v_{f}$ be two positive numbers, $v_{s}<v_{f}$. Furthermore, let us denote by $\mathcal{S}(r, P)$ the circle with radius $r$ centered at $P$, and by $\mathcal{B}(r, P)$ the open disk with radius $r$ centered at $P$.

Definition 1. Let $C$ and $C_{O}$ be points on the plane and $R_{O}<R<R_{B}, R_{k}=$ $R v_{s} / v_{f}$ be positive numbers for which $R-R_{O}$ is greater than the distance of $C$ and $C_{O}$. The circle $O:=\mathcal{S}\left(R_{O}, C_{O}\right)$ is called obstacle, $I:=\mathcal{S}(R, C)$ is called interface (or interfacial boundary), $B:=\mathcal{S}\left(R_{B}, C\right)$ is called (outer) boundary, $K:=\mathcal{S}\left(R_{k}, C\right)$ is called caustic. Let $S:=\mathcal{B}(R, C) \backslash \mathcal{B}\left(R_{O}, C_{O}\right)$ be called slow region, $F:=B \cup \mathcal{B}\left(R_{B}, C\right) \backslash \mathcal{B}(R, C)$ be called fast region and $V:=S \cup F$ be called reactor. (See Fig. 1.)

Assumption 1. We assume that $\mathcal{B}\left(R_{O}, C_{O}\right) \cap \mathcal{B}\left(R_{k}, C\right)=\emptyset$ (strong asymmetry).

In this case the circles $O$ and $K$ have two pairs of common tangents: the external common tangents, and the internal common tangents. Denote the point of $O$ which is the nearest to $K$ by $O_{K}$. The tangent points on $O$ with the four common tangents are $O_{d}, O_{a}, O_{b}$, and $O_{c}$ ordered in the anticlockwise direction starting from $O_{K} \cdot K_{d}, K_{b}$, $K_{a}$, and $K_{c}$ will denote the corresponding tangent points in $K$ with the same common 
tangents, respectively. The common tangents have 8 endpoints on $I$, namely $I_{d}, I_{a}, I_{b}$, $I_{c}$, as well as $I_{d}^{\prime}, I_{b}^{\prime}, I_{a}^{\prime}, I_{c}^{\prime}$. (Those farther from $O$ are primed, see Fig. 1.)

Each of the four common tangents is divided into three segments by the obstacle and the caustic:

- $I_{a} I_{a}^{\prime}=a \cup a^{\prime \prime} \cup a^{\prime}$ where $a=I_{a} O_{a}, a^{\prime \prime}=O_{a} K_{a}$ and $a^{\prime}=K_{a} I_{a}^{\prime}$;

- $I_{b} I_{b}^{\prime}=b \cup b^{\prime \prime} \cup b^{\prime}$ where $b=I_{b} O_{b}, b^{\prime \prime}=O_{b} K_{b}$ and $b^{\prime}=K_{b} I_{b}^{\prime}$;

- $I_{c} I_{c}^{\prime}=c \cup c^{\prime \prime} \cup c^{\prime}$ where $c=I_{c} O_{c}, c^{\prime \prime}=O_{c} K_{c}$ and $c^{\prime}=K_{c} I_{c}^{\prime}$;

- $I_{d} I_{d}^{\prime}=d \cup d^{\prime \prime} \cup d^{\prime}$ where $d=I_{d} O_{d}, d^{\prime \prime}=O_{d} K_{d}$ and $d^{\prime}=K_{d} I_{d}^{\prime}$.

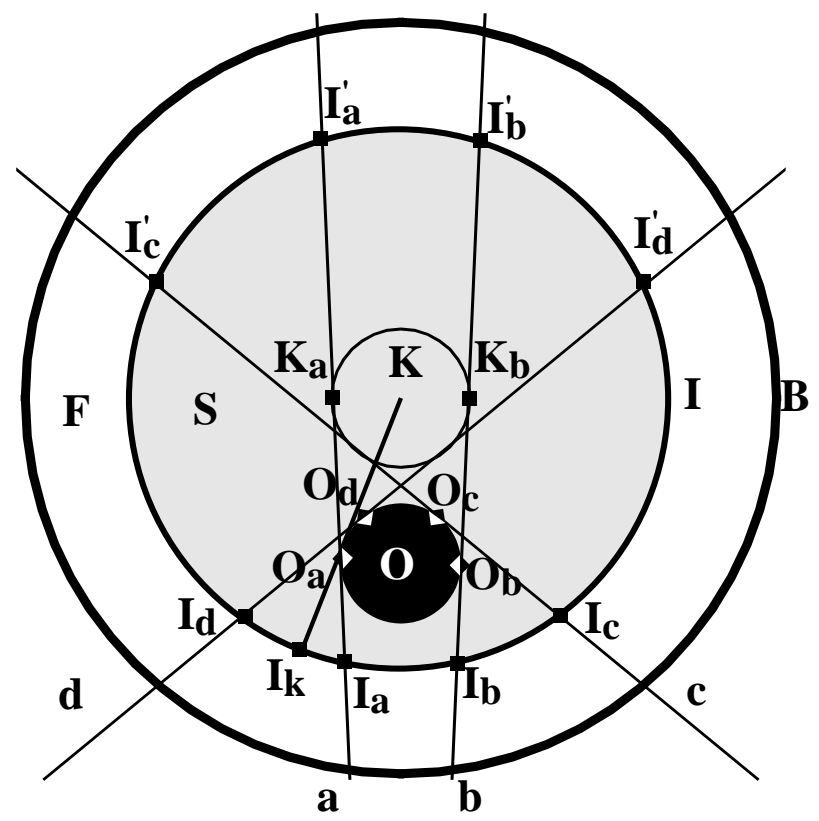

Fig. 1. Strongly asymmetric arrangement. Illustration of notation:

$K$ : caustic, $O$ : obstacle, $I$ : interface, $B$ : outer boundary, $S:$ slow region (gray),

$F$ : fast region, $a, b:$ common outer tangents, $c, d:$ common inner tangents,

$k$ : tangent line drawn from the center of $K$ to $O$. The notation of common points of two curves indicates both curves, e.g. $I_{a}$ and $I_{a}^{\prime}$ are common points of $I$ and $a$.

Definition 2. Let the velocity $v: V \rightarrow \mathbf{R}$ be the function

$$
v(x):= \begin{cases}v_{s} & \text { if } x \in S \\ v_{f} & \text { if } x \in F .\end{cases}
$$

Remark that if a part of the path lies on the interfacial boundary, then we should assign the greater velocity for that part due to Fermat's principle.

Definition 3. Let $f_{0} \in V$ be a straight segment, the endpoints of which are on $O$ and $B$, respectively; this will be referred to as initial front.

The assumption that $f_{0}$ is a straight segment is only a technical condition. 
Definition 4. Let $G$ be the set of piecewise smooth curves in $V$ for which the following two conditions hold:

- The starting point of the curve is on $f_{0}$.

- The curve can intersect $f_{0}$ only in positive direction, that is, a small arc of the curve after the intersection point is on the local positive side. (The points on the local positive side can be obtained from the points of $f_{0}$ by a small anticlockwise rotation around $C_{O}$.)

These curves will be called admissible curves.

The second condition in the above definition ensures that starting from the initial front the propagation is allowed only in the anticlockwise direction.

Definition 5. Let $g \in G$ be an admissible curve given in arc length parametric form; the length of $g$ is denoted by $l(g)$. Let us denote by $\tau(g)$ the propagation time along $g$, that is,

$$
\tau(g)=\int_{0}^{l(g)} \frac{1}{v(g(s))} d s .
$$

Definition 6. A piecewise smooth curve in $V$ is called a Fermat ray or extremal, if there is no other piecewise smooth curve with same endpoints having less propagation time.

Until now Fermat rays belonged to two points, now we define Fermat rays belonging to a front.

Definition 7. Let $f$ be a piecewise continuous curve in $V$. The Fermat rays starting from $f$ are such Fermat rays for which their starting point are on $f$ and there is no other Fermat ray with the same endpoint having less propagation time.

The Fermat rays starting from $f$ are orthogonal to $f$ (transversality condition for the extremals).

Definition 8. A simple closed curve surrounding the obstacle in $V$ is called a minimal loop if there is no other closed curve surrounding the obstacle $O$ having less propagation time.

THEOREM 1. The minimal loop consists of Fermat rays.

Proof. If a part of the minimal loop were not a Fermat ray, we could substitute that part by a Fermat ray with the same endpoints. This way we could construct a loop with shorter propagation time, which is a contradiction.

Definition 9. The mixed loop $M$ is the convex closed curve consisting of the circular $\operatorname{arc} O_{b} O_{a}$ on $O$, the circular arc $I_{a} I_{b}$ on $I$ and the connecting segments $O_{a} I_{a}, I_{b} O_{b}$ of the external common tangents.

The mixed loop does not exist if the segments $a$ and $b$ (Fig. 1) have intersection point in $S$.

TheOREM 2. The minimal loop is either $O$, or $I$, or $M$.

Proof. Obviously, if the minimal loop has no point inside $S$, then the minimal loop is either $O$ or $I$. On the other hand, if it has a point $P$ in $S$ then the arc of the minimal 
loop containing $P$ is a line segment, since the minimal loop must consist of Fermat rays. The connection of this line segment with $I$ must be with the critical angle of total reflection, and the connection to $O$ must be tangential according to the junction rules [12]. Consequently, this line must be common tangent to $O$ and $K$. The internal common tangents cannot be parts of the minimal loop. Suppose the contrary: then a contradiction would arise at the joining at $I$, since the ray coming from $O$ would turn at $I$ at the "bad" direction, the loop would not encircle $O$. Since the rays departing from $I$ are Fermat rays until they reach $K$, it is not possible to interconnect two points of $I$ through $P$ with a Fermat ray. Thus the arc must have an endpoint on $I$ and the other one on $O$. As a consequence of this the arcs of the minimal loop are on $I, O$ and the common tangents of $O$ and $K$. Inside $S$ only the following line segments which connect $I$ and $O$ are Fermat rays: $I_{d} O_{d}, I_{a} O_{a}, I_{b} O_{b}, I_{c} O_{c}$ (Fig. 1). From this, any closed curve using $I_{d} O_{d}$ or $I_{c} O_{c}$ lines with correct arc connections would exclude the obstacle. Finally, the only remaining possibility is the closed convex curve $I_{a} I_{b} O_{b} O_{a}$, that is the mixed loop $M$. The wave propagation in the geometric wave theory is determined by Fermat's principle, therefore the fronts are defined as the level curves of the following eikonal.

Definition 10. Let $\Sigma: V \rightarrow R+$ be the eikonal, that is

$$
\Sigma(P)=\inf \{\tau(g): g \in G, g(l(g))=P\} .
$$

Let the wave front at time $t$ be

$$
f_{t}=\{P \in V: \Sigma(P)=t\} .
$$

The aim of this paper is to determine the shape and evolution of the fronts $f_{t}$. It turns out that after a transient interval, the process will be periodic in time with period $T$, that is an 'asymptotic' or 'permanent' front portrait. Its fronts rotate around the obstacle periodically, during a round the shape of the front changes (not as in the symmetric case), but for the case of simplicity we refer to these fronts as 'permanent' fronts.

Our aim is to determine this permanent front portrait. The fronts are orthogonal to the extremals, therefore in order to get the shape of the fronts it is enough to determine the extremals. In our case the Fermat rays consist of arcs. There are two types of arcs: interior arcs, which are straight segments, and boundary arcs, which lie on the interfacial boundary or on the obstacle. The rules of their connections (junction rules) were derived from the Fermat principle: two adjacent arcs can be joined together according to the laws of refraction. Interior arcs in different media are attached according to Snell's law. Interior and boundary arcs can be connected in two ways: tangentially (toward the faster region) or with the critical angle of total reflection (toward the slower region).

In the following we use the term reverse involute in the case when the center of the involute's osculating circle is before the front. The permanent wave fronts in our case are made of involute parts of the obstacle, reverse involute parts of the caustic $(K)$ in region $S$, while in region $F$ they are made of involute parts of the interfacial boundary and involute parts of caustic ( $K 2$, see below).

The caustic $K$ (Definition 1 and Fig. 1) is the envelope of the rays departing from the interfacial boundary into the slower region with the critical angle of total reflection, 
while the caustic $K 2$ is the envelope of the refracted rays.

While determining the extremals, certain points of the fronts (the "leading points") play a distinguished role. The permanent wave portrait is determined by the leading point which moves along the minimal loop. It strongly depends on the relative position of the obstacle and the caustic.

Definition 11. The point $P$ of the front $f_{t_{0}}$ is called a leading point if for a certain $t>t_{0}$ there exists a positive measure set of points $Q \in f_{t}$ for which there exists an extremal starting from $P$ at $t_{0}$, ending in $Q$ at $t$.

Usually a point of a front generates a single point of each subsequent front. A leading point is exceptional: it generates a finite front part.

Definition 12. A front loop is a closed part of the front, and along it the front moves inside.

3. Break point dynamics. To study the evolution of fronts we first consider the "break point dynamics". Break point is used in the same sense as in the previous paper [12]. After a certain transient period some parts of initial front will disappear. Only the front components generated by leading points will survive. So the entire front will be generated by rays emanated from the leading points running along $O$ and $I$, respectively.

There are eight elementary families of rays, four of them are related to $O$ and four are related to $I$ :

$\mathrm{SO}+$ : the family of straight rays departing tangentially from a leading point on $O$ running in the anticlockwise direction, the generated fronts are involutes of $O$ moving anticlockwise,

$S O-$ : the family of straight rays departing tangentially from a leading point on $O$ running in the clockwise direction, the generated fronts are involutes of $O$ moving clockwise,

$\mathrm{FO}+$ : the rays belonging to $S O+$ refract at $I$, according to Snell's law, and those refracted rays constitute the family $\mathrm{FO}+$

$F O-$ : the rays belonging to $S O$ - refract at $I$, according to Snell's law, and those refracted rays constitute the family $\mathrm{FO}-$,

$S I+$ : the family of straight rays departing (with the critical angle of total reflection) from a leading point running on the interface $I$ in anticlockwise direction, the generated fronts are reverse involutes of $K$ moving anticlockwise,

$S I-$ : the family of straight rays departing (with the critical angle of total reflection) from a leading point running on the interface $I$ in clockwise direction, the generated fronts are reverse involutes of $K$ moving clockwise,

$F I+$ : the set of rays emanated tangentially from the leading point running on $I$ in the anticlockwise direction respectively, the generated fronts are involutes of $I$ moving anticlockwise,

$F I-$ : the set of rays emanated tangentially from the leading point running on $I$ in the clockwise direction respectively, the generated fronts are involutes of $I$ moving clockwise. 
The same notation will be applied to the corresponding front components (a front is composed of one or more parts, each part is generated by rays of a given family of rays, and we refer to these parts as front components).

A break point separates parts of a front generated by these two leading points, or more precisely, generated by rays from two different "elementary families of rays" belonging to different leading points. In contrast, a refraction point separates parts of a front generated by rays from two different "elementary families of rays" belonging to $O$. Along a particular front there may be three different types of singular points, where the tangent is discontinuous: break points $(S O+S I+, S O-S I+, S O+S I-, S O-S I-, F O+F I+$, $\mathrm{FO}-\mathrm{FI}+\mathrm{FO}+\mathrm{FI}-$ and $\mathrm{FO}-\mathrm{FI}-$ ), refraction points $(\mathrm{SO}+\mathrm{FO}+, \mathrm{SO}-\mathrm{FO}-)$ and leading points $(S I+F I+, S I-F I-$ and on $O)$.

In the evolution of fronts three different stages can be distinguished:

- first transient stage,

- second transient stage,

- periodic stage.

The first transient stage terminates when the entire front is generated by rays from the above eight elementary families. During the second transient stage the competition of leading points takes place. As a result of this competition the final permanent wave fronts are established, and the process becomes periodic in time (periodic stage).

In this paper the break points will be labeled with the two joining front components, e. g. break point $S O+S I+$ separates an obstacle involute and a caustic reverse involute, both rotating anticlockwise.

3.1. Virtual break point dynamics and the bisector rule. In the previous work it turned out that break points are important when studying the evolution of wave fronts. At the break points the tangent of the front is discontinuous. Let us imagine an arbitrary piecewise smooth front. Any break point within a homogeneous medium moves along the bisector of the left-side and right-side tangents joining at the break point (bisector rule, Fig. 2). Recall that not only the direction but also the magnitude of the velocity yields from the bisector rule [12].

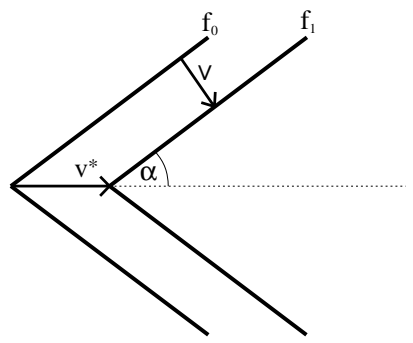

Fig. 2. The bisector rule in homogeneous medium. The break point of a concave front $f_{0}$ moves along the bisector with the velocity $v^{*}=v / \sin \alpha$, where $v$ is the wave velocity and $2 \alpha$ is the angle at the break point.

Now the concept of break point needs a slight refinement. It seems reasonable to include such points where the tangent is continuous, but the front part at one side is 
generated by rays from an elementary family of rays which differs from the elementary family generating the front part at the other side of the break point. So, the break point in this new sense is determined by two elementary families of rays, and the break point is labeled by them. The term 'virtual break point' is used in such sense.

Definition 13. $\{P,(A, B)\}$ is called a virtual break point, where $P \in V \backslash I$ and $(A, B)$ is a pair of elementary families of rays with the requirements

- if $P \in S$ then the first index of $A$ and $B$ must be $S$,

- if $P \in F \backslash I$ then the first index of $A$ and $B$ must be $F$.

Remark that the order of $A$ and $B$ is not significant: $\{P,(A, B)\}=\{P,(B, A)\}$.

Definition 14. $\{P,(A, B)\}$ is called a refraction point if $P \in I$ and

- $(A, B)=(S O+, F O+)$

- or $(A, B)=(S O-, F O-)$.

Remark that $\{P(A, B)\}$ is a leading point on $I$ if $P \in I$ and $(A, B)=(S I+, F I+)$ or $(A, B)=(S I-, F I-)$.

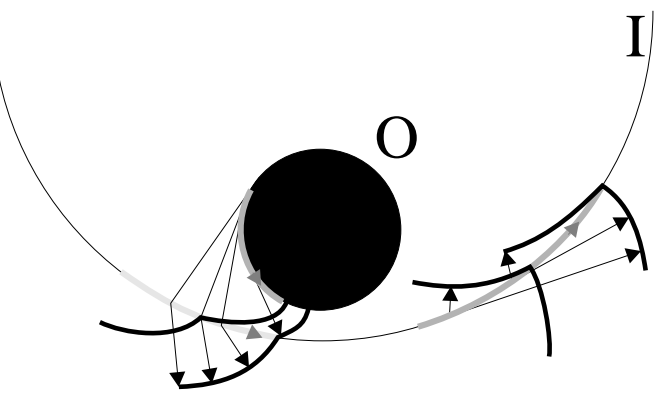

Fig. 3. Leading points (medium gray), refraction points (light gray) and the corresponding rays and fronts.

In typical cases the front is not smooth at virtual break points, but smooth connection can also occur. Applying the bisector rule, we can compute the velocity of any virtual break point at any inner point of the reactor. To construct the "velocity field" we have to consider that through each point two wave front parts of different type pass and then apply the bisector rule. This way we can associate a velocity field to any given class of virtual break points, that is different virtual break point dynamics yield. Those dynamics and their velocity fields will be labeled in the same way as the classes of virtual break points, namely by a pair of elementary families of rays. Using virtual break point dynamics we can follow the evolution of a front locally in the vicinity of virtual break points.

The introduction of virtual break points at each point of the reactor helps us to understand the qualitative motion of the actual break points, especially at the permanent stage. By applying the bisector rule to the motion of the virtual break point, the velocity field inside $S$ can be computed. Not like in the previous paper, now six different types of break point exist in $S$, those imply six different velocity fields.

The dynamics of the break points $S I+S I-$ is very simple: the bisector rule shows that such break points always move inward to the center $C$. Similarly, the break points 
$S O+S O$ - always move outward from the center $C_{O}$.

Fig. 4 shows the velocity fields of the break points $S O+S I+$ and $S O+S I-$, respectively. In this figure the length of the arrows is proportional to $1+\arctan \left(v^{*}-v_{s}\right)$ (where $v^{*}$ is the velocity of the break point): we used this transformation to avoid the difficulties arising from the infiniteness of the velocity at certain points.
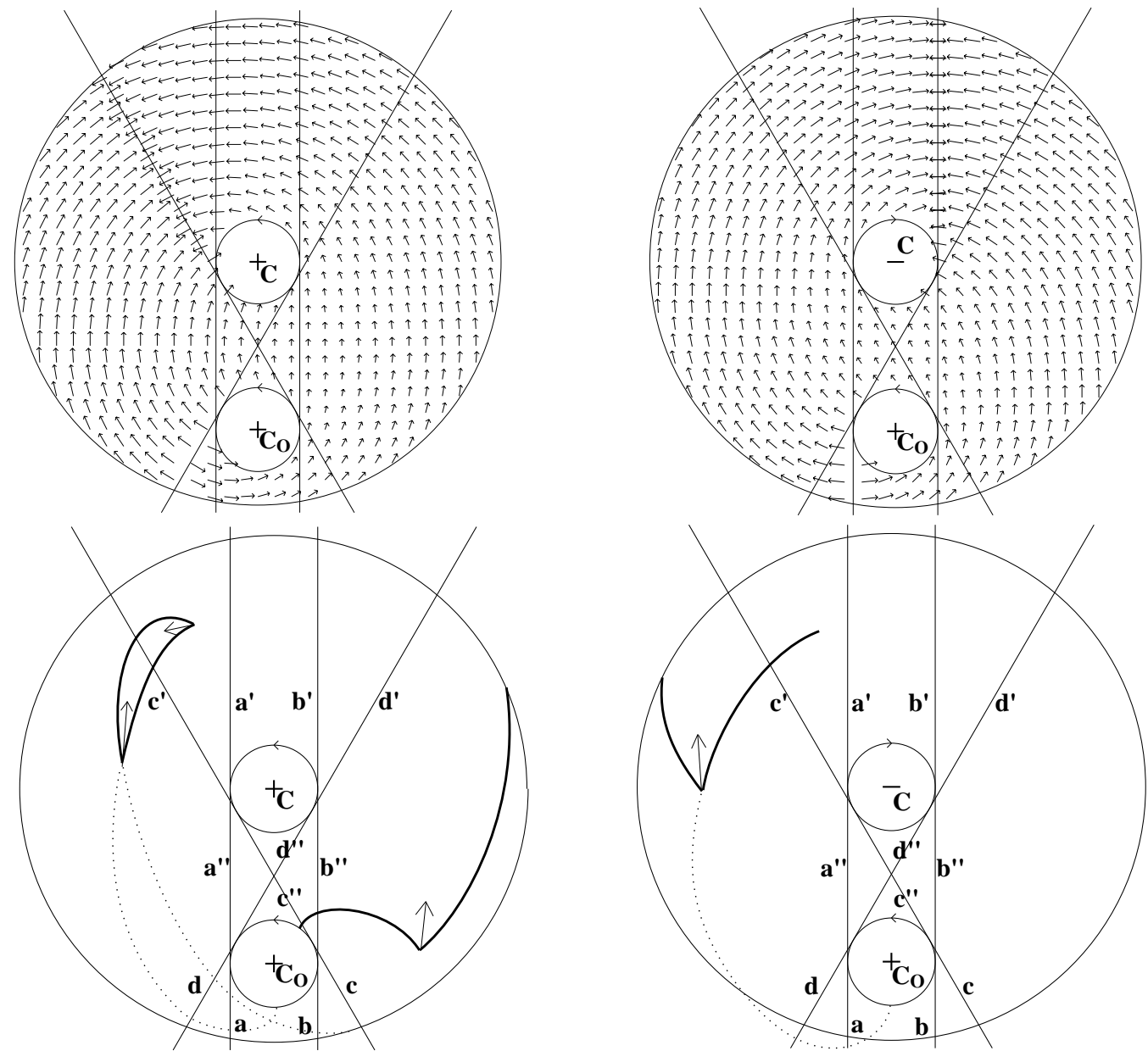

Fig. 4. The velocity fields of the virtual break points $S O+S I+$ (on the top left) and $S O+S I-$ (on the top right). The two bottom figures illustrate the connection between the break point velocity vector and the corresponding front parts.

The dotted lines are continuations of the front parts.

Finally, the velocity field for $S O-S I-$ is the reflection (with respect to the axis $C C_{O}$ ) of that for $S O+S I+$, and similarly, the velocity field for $S O-S I+$ is the reflection of the one for $S O+S I-$.

For the case $S O+S I+$, in a domain limited by the inner tangent segments $c^{\prime}$ and $d$, the arc $I_{c}^{\prime} I_{d}$ and the segment $K_{c}^{\prime} O_{d}$ the break point moves opposite to the motion of the leading points. Along the segments $c^{\prime}$ and $d$ the angle of the two Fermat rays is $\pi$, so 
there is no unique direction of the bisector (two opposite directions are possible), and the break point velocity is infinite, so the points on these segments are singular. If we consider the neighborhood of these common tangents, it turns out that the break points depart from $d$ and approach to $c^{\prime}$. It means that in one point of $d$ the involute and the reverse involute collide, and two new break points arise. In any point of $c^{\prime}$ some break points belonging to a front loop cease to exist.

The break point velocity has its minimum (with the minimum value $v_{s}$ ) when the two Fermat rays have the same direction. In these points an $S O+S I+$ break point can be born. Now these points are on $b^{\prime \prime}$.

Similarly, for a break point of the type $S O+S I-$, the break point velocity is infinite along $a$ and $b^{\prime}$. The break points depart from $a$ and approach to $b^{\prime}$. The break point velocity is minimal on $c^{\prime \prime}\left(v=v_{s}\right)$.

3.2. Permanent wave portraits. As was stated formerly, after a transient period, permanent wave fronts move around the obstacle. These permanent fronts establish as a result of competition of leading points running along $O$ and $I$, respectively. These leading points have their own regions in which the wave fronts are generated by rays emanating from them.

Earlier we introduced the concept of the elementary families of rays. In this section we restrict this concept to the permanent rays. The elementary families of permanent rays contain only the rays which really exist on the permanent wave portrait. The permanent wave fronts divide the medium into zones: one zone belongs to one elementary family of permanent rays. The permanent rays cannot go beyond the border of the relevant zone.

The borders of the zones are the conflict sets of certain previous fronts, or, on other hand, they are the Voronoi diagrams of the two competing leading points running along the obstacle and along the interface, respectively, see e.g. [2, 6, 8, 9]. The zones themselves are territories of those previous fronts or of the moving leading points.

To describe permanent waves qualitatively we should determine the structure of zones. The borders of zones consist of parts of break point trajectories, arcs of the obstacle, of the interface, or of the outer boundary, respectively. To get information about the zone structure, we investigate the creation and cessation of break points.

3.3. Appearance of break points. One break point can be born in a point of $O$ or $I$, where two Fermat rays belonging to different families of rays coincide. Such points on $I$ are $I_{a}$ and $I_{d}$, where from an arriving $F O+($ and $S O+$ ) front component, break points $\mathrm{FI}+\mathrm{FO}+, \mathrm{SI}+\mathrm{SO}+($ and $\mathrm{FI}-\mathrm{FO}+, \mathrm{SO}+\mathrm{SI}-$, respectively) can be born, and also a new $F I+(F I-)$ front component can appear (Fig. 5, subclass O3). On the other hand, on the obstacle at point $O_{d}$ and $O_{b}$, from an arriving $S I+$ front component, a break point $S O+S I+($ and $S O-S I+$, respectively) can be born, and also a new $S O+(S O-)$ front component can appear.

Two break points can be born when two parts of the same front collide frontally. In this case the two newly born break points move to the opposite direction, and the Fermat rays belonging to the colliding fronts must also have opposite direction in the point of the collision. 
In the strong asymmetric arrangement two leading points can collide frontally on the interface, and after the collision there will be two new break points moving perpendicular to $I$, one of them moves in $S(S I+S I-)$ and the other in $F(F I+F I-)$ (Fig. 5, subclass O3). Two leading points can collide also on $O$ in a similar manner, except that in this case only one new break point can appear $(S O+S O-)$, and it moves outward perpendicular to $O$ (Fig. 5, subclass I3-2).

On the interface a leading point also can collide with a refraction point. In this case there must be a break point $S O+S I+$. When such points on $I$ collide, this break point also arrives to $I$, and after that the break point changes its type to $F O+F I+$, and departs from $I$ in $F$ (Fig. 5, subclass O0).

3.4. Collision and disappearance of break points. The simplest way for one break point to disappear is a collision with the obstacle or with the outer boundary. Collision of two adjacent break points assumes three different parts in the actual front. The break points collide in a point where the middle front component disappears, so only one break point remains, that is, the two break points merge.

Two break points can collide frontally (with an angle $\pi$ ) only if they are in a front loop (Fig. 5, subclasses I2, O2). A simple type of front loops consists of only two front components, and it contains only two break points. Such a front loop can be born when two front components collide in a point of the common tangent $d$. Since the front moves inward the loop, the loop will be smaller and smaller until it vanishes, and the two break points collide frontally. In this critical point the direction of the break point velocity is not unique, so there must be a singularity of the break point velocity field inside such diminishing front loop. This singularity occurs only at points of $c^{\prime}$.

In our case there is another type of front loops, which consists of three front components, and so they contain three break points (Fig. 5, subclass O3). While such a loop is diminishing, two of its break points can collide, and it becomes a simple two-edged loop. However, as we can see, there is another possibility: when the three break points collide and disappear simultaneously.

3.5. Leading points on $I$ and $O$. The following consideration aims to determine the possible behavior of the permanent wave propagation along the interface.

Recall that a front component is labeled by three characters which will be called indexes in the following. The first index refers to the region $(S$ or $F$ ), the second one refers to the generating leading point $(O$ or $I)$, and the third one gives the direction of rotation $(+$ or -$)$.

At the interface two front components join, the first index of the one is $S$, that of the other is $F$. Their third index must be identical, it characterizes the propagation direction of the wave near the interface. The virtual velocity of the two joining front components should be equal [12], consequently the second index also must be the same for the joining front components. Otherwise, in asymmetric case the equality of the two virtual velocities can occur only at few isolated points. Therefore, only the following four possibilities remain: $S O+F O+, S O-F O-, S I+F I+, S I-F I-$. The first two fronts contain a refraction point, the latter ones contain a leading point at the interface. 
In addition to the above exclusion rules, certain parts of the interface are forbidden for some type of fronts. If the virtual velocity of an obstacle generated front reduces to the velocity $v_{f}$, then a leading point appears, or, in other words, the refraction point changes into a leading point running along the interface. This transformation occurs when the ray $S O+$ reaches the interface at the critical angle of total refraction, that is, at the points $I_{a}, I_{d}$, and $I_{b}^{\prime}, I_{c}^{\prime}$, respectively (Fig. 1). Therefore, the $\operatorname{arcs}\left(I_{a}, I_{b}^{\prime}\right)$, and $\left(I_{c}^{\prime}, I_{d}\right)$ are forbidden to the fronts $\mathrm{SO}+\mathrm{FO}+$. (It is easy to see that the $\mathrm{SO}$ - front part cannot reach the obstacle.)

\begin{tabular}{|c|c|}
\hline Arc & Possible front pairs \\
\hline \hline$\left(I_{a}, I_{b}\right)$ & $S I+F I+$ \\
\hline$\left(I_{b}, I_{d}^{\prime}\right)$ & $S I+F I+$ \\
\hline$\left(I_{d}^{\prime}, I_{b}^{\prime}\right)$ & $S I+F I+, S I-F I-$ \\
\hline$\left(I_{b}^{\prime}, I_{c}^{\prime}\right)$ & $S I+F I+, S I-F I-, S O+F O+$ \\
\hline$\left(I_{c}^{\prime}, I_{d}\right)$ & $S I+F I+, S I-F I-$ \\
\hline$\left(I_{d}, I_{a}\right)$ & $S I+F I+, S O+F O+$ \\
\hline
\end{tabular}

Table 1. Leading points on $I$.

From the two possible directions of rotating waves let us choose the anticlockwise $(+)$. For this case the following possible leading or refraction points occur in $I$ (Table 1 ).

If the minimal loop is the interface, then the leading point $S I+F I+$ runs on the entire interface. The details for the other possible cases are discussed later.

\begin{tabular}{|c|c|}
\hline Arc & Possible fronts \\
\hline \hline$\left(O_{b}, O_{K}\right)$ & $S O+$ \\
\hline$\left(O_{K}, O_{d}\right)$ & $S O+, S O-$ \\
\hline$\left(O_{d}, O_{b}\right)$ & $S O+, S I+$ \\
\hline
\end{tabular}

Table 2. Front parts on $O$.

Considering the leading point on the obstacle is simpler. At the obstacle there can be only three types of fronts: $S O-, S O+, S I+$. (Remark that $S I-$ cannot occur at the obstacle for waves rotating anticlockwise.) The endpoints of the first two fronts are leading points on the obstacle. The virtual velocity of the interface generated front $S I+$ on the obstacle can never be smaller than $v_{s}$. The two velocities are equal at the points $O_{b}$ and $O_{d}$. If the minimal loop is the obstacle, then $S O+$ front runs along the entire obstacle. Otherwise, at some parts of the obstacle there is a front $S I+$, and a leading point on $O$ running along the positive direction appears at $O_{b}$. Under certain conditions a leading point on $O$ running along the negative direction appears at $O_{d}$. The appearance of leading point on the obstacle is due to the fact that $O$ shadows the rays coming from $I$ in certain regions.

All the possible cases are listed in Table 2. 


\section{Classification of permanent wave portraits}
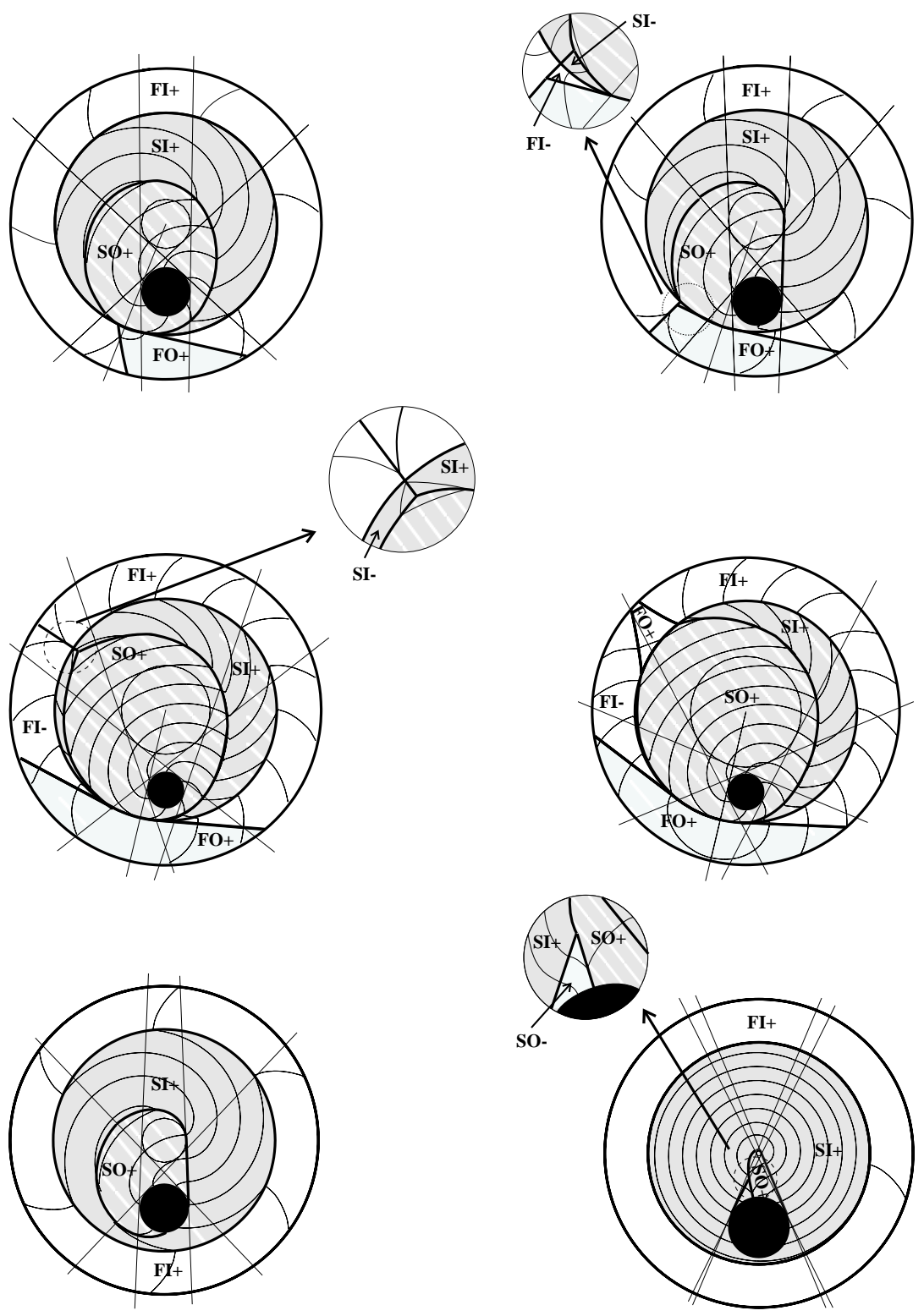

Fig. 5. The maps of the subclasses: O2, M3-2, O3, O0, I2, I3-2. The fronts are thin lines, the borders of the zones (break point, leading point and refraction point trajectories) are thick lines.

Construction of wave portraits and zones. In the investigation of the permanent wave portraits and in their classes, the creation and cessation of break points plays an important role. It is also necessary to follow the fate of the newly born break points, their motion and possibly collisions during a whole round. The trajectories of the break points 
are governed by break point dynamics, and they mark the borders of the zones. From the map of zones the stationary fronts can be drawn.

Classification. As was shown in the previous paper [12], the minimal loop plays a key role in the permanent wave portraits. There are three types of minimal loops (obstacle, mixed and interface), the corresponding three classes of permanent wave portraits will be labeled by the letters $\mathrm{O}, \mathrm{M}$ and I, respectively.

The classes $\mathrm{O}$ and $\mathrm{M}$ have four, the class I has two subclasses based on the topology of the zone structure (shortly map) determined by the layout of the permanent fronts. The number of the front components in the front loop characterizes subclasses: we use this number to label the subclasses. Number 0 stands for the case when there is no loop in the fronts. Number 1 cannot occur. In certain subclasses a three-arced loop reduces to a two-arced one, we use the symbol 3-2 for those subclasses.

Class $\mathrm{O}$ (minimal loop is the obstacle). In this case there is a leading point moving forward along the whole $O$, so the zone $S O+$ engulfs $O$ totally. At the point $I_{a}$ two break points and a leading point appear: a break point $S O+S I+$, which moves inward, and an $F I+F O+$, which moves outward tangentially from the interface, and a $S I+F I+$ leading point, which runs along the interface (Fig. 5, subclasses O2, O3, O0). This anticlockwise moving leading point cannot reach $I_{a}$, on the remaining part of the interface there is a refraction point or a clockwise moving leading point (the details of its trajectory are discussed in the different subclasses).

Subclass O2. The front loop is born by colliding of the obstacle involute and the caustic reverse involute in a point of $d$ (Fig. 1), and this loop is always two-edged until its cessation. In the point of collision two new break points appear: one of them goes through the interface and reaches the outer boundary; and the second turns backward, while it collides with the break point appearing in $I_{a}$ at a point of $c^{\prime}$ (Fig. 1), and then the front loop disappears.

Subclass O3-2. The obstacle involute reaches the $I_{d}$ point, where two new break points $(S O+S I-$ and $F I-F O+)$ and a backward moving leading point appear in a manner similar to the point $I_{a}$, but in the opposite direction. Later on the interface two leading points collide frontally, and then two break points $(S I-S I+$ and $F I-F I+)$ appear. In the moment of the collision a three-edged front loop is born. This takes place in the $I_{c}^{\prime} I_{d}$ arc of the interface. Later the backward moving caustic reverse involute vanishes, when the break points $S O+S I-$ and $S I-S I+$ collide, so the front loop contains only two front components when it disappears at a point of $c^{\prime}$ like in subclass O2. When the outer boundary is far enough, the break points $F I-F I+$ and $F I-F O+$ can collide, and so an $F O+F I+$ break point remains.

Subclass O3. If the backward moving leading point on $I$ passes $I_{c}^{\prime}$, then it collides with the other leading point in $I$ at a certain point of the arc $I_{b}^{\prime} I_{c}^{\prime}$, and a three-edged front loop is born, like in subclass O3-2. This loop disappears by the colliding of its three break points simultaneously.

Subclass O0. This case can occur if the break points $S O+S I-\left(\operatorname{arising}\right.$ in $\left.I_{d}\right)$ and $S O+S I+\left(\operatorname{arising}\right.$ in $\left.I_{a}\right)$ reach the interface again before the corresponding leading points 
collide on $I$. Between these leading points one or two $S O+F O+$ refraction points move on $I$.

Class M (minimal loop is the mixed one). In this case the obstacle involute arrives at $I_{a}$ similarly to the previous case, but the break point $S O+S I+$ collides with the boundary of the obstacle before $O_{b}$. So a caustic reverse involute arrives at the point $O_{b}$, where a new $S O+S I+$ break point moving along $b^{\prime \prime}$, and a leading point moving anticlockwise on $O$ arise. The behavior of the front is the same as in the previous case between points $O_{b}$ and $I_{a}$, so there are the same subclasses for this class as those in the class O. As a contrast to class $\mathrm{O}$, now the leading point on $O$ cannot exist on the whole circle $O$.

Class I (minimal loop is the interface). If the minimal loop is the interface, a leading point runs all over the whole $I$. The caustic's reverse involute reaches the point $O_{b}$, where an $S O+S I+$ break point and a leading point on $O$ arise. The obstacle involute cannot reach the interface, so no break points can appear on $I$.

Subclass I2. A two-edged front loop arises when the obstacle involute collides with the caustic reverse involute in a point of $d$. In that point of $d$ two new $S O+S I+$ break points appear: one of them reaches the boundary of the obstacle before the point $O_{b}$; and the other one moves backward, until it collides with the break point coming from $O_{b}$ at a point of $c^{\prime}$, and then the front loop disappears.

Subclass I3-2. The caustic reverse involute touches the obstacle, and then it splits. The left part of that involute reaches the point $O_{d}$, where a backward moving $S O-S I+$ break point and a backward moving leading point on $O$ appear in a manner similar to the point $O_{b}$, but in the opposite direction. The two leading points on the boundary of $O$ collide frontally, and then one $S O-S O+$ break point is born. In the moment of the collision a three-edged front loop arises. Later the backward moving involute of the obstacle vanishes, when the break points $S O-S O+$ and $S O-S I+$ collide, after that the front loop contains two front components until it disappears at a certain point of $c^{\prime}$.

Acknowledgments. The authors thank Dirk Siersma and Martijn van Manen for consultation. A. Volford thanks for the Békésy György Postdoctoral Fellowship. This work was partially supported by OTKA grants T-30110 and T-042708.

\section{References}

[1] G. Albers, L. J. Guibas, J. S. B. Mitchell, T. Roos, Voronoi diagrams of moving points, Internat. J. Comput. Geom. Appl. 8 (1998), 365-379.

[2] J. W. Bruce, P. J. Giblin, C. G. Gibson, Symmetry sets, Proc. Roy. Soc. Edinburgh Sect. A 101 (1985), 163-186.

[3] J.-J. Fu, R. C. T. Lee, Voronou diagrams of moving points in the plane, Internat. J. Comput. Geom. Appl. 1 (1991), 23-32.

[4] K. Kály-Kullai, A fast method to simulate travelling waves in nonhomogeneous chemical or biological media, J. Math. Chem. 34 (2003), 163-176. 
[5] A. Lázár, H. D. Försterling, H. Farkas, P. L. Simon, A. Volford, Z. Noszticzius, Waves of excitations on nonuniform membrane rings, caustics, and reverse involutes, Chaos 7 (1997), 731-737.

[6] M. van Manen, Curvature and torsion formulas for conflict sets, this volume, 209-222.

[7] J. Sainhas, R. Dilão, Wave optics in reaction-diffusion systems, Phys. Rev. Lett. 80 (1998), $5216-5219$.

[8] D. Siersma, Voronoi diagrams and Morse theory of the distance function, in: Geometry in Present Day Science, O. E. Barndorff-Nielsen and E. B. V. Jensen (eds.), World Scientific, Singapore, 1999, 187-208.

[9] D. Siersma, Properties of conflict sets in the plane, in: Geometry and Topology of CausticsCaustics '98, Banach Center Publ. 50, Warszawa, 1999, 267-276.

[10] P. L. Simon, H. Farkas, Geometric theory of trigger waves - a dynamical system approach, J. Math. Chem. 19 (1996), 301-315.

[11] J. Sotomayor, D. Siersma, R. Garcia, Curvatures of conflict surfaces in Euclidean 3-space, in: Geometry and Topology of Caustics - Caustics '98, Banach Center Publ. 50, Warszawa, 1999, 277-285.

[12] A. Volford, P. L. Simon, H. Farkas, Waves of excitations in heterogeneous annular region, asymmetric arrangement, in: Geometry and Topology of Caustics-Caustics '98, Banach Center Publ. 50, Warszawa, 1999, 305-320.

[13] A. Volford, P. L. Simon, H. Farkas, Z. Noszticzius, Rotating chemical waves: theory and experiments, Physica A 274 (1999), 30-49.

[14] N. Wiener, A. Rosenblueth, The mathematical formulation of the problem of conduction of impulses in a network of connected excitable elements, specifically in cardiac muscle, Arch. Inst. Card. de Mexico 16 (1946), 205-265. 